\title{
Quality over quantity: Achieving Better Resolution in Subtomogram Averaging Using Less particles
}

Beata Turonova ${ }^{1}$, Shyamal Mosalaganti ${ }^{1}$ and Martin Beck ${ }^{2}$

${ }^{1}$ European Molecular Biology Laboratory, Heidelberg, Baden-Wurttemberg, Germany, ${ }^{2}$ Max Planck Institute of Biophysics, Frankfurt am Main, Hessen, Germany

Cryo-Electron Tomography (cryoET) is an important technique in modern structural biology, as it enables us to study cellular ultrastructure and large macromolecular complexes in close to their native environment. In combination with subtomogram averaging (SA) we can now resolve structures in great detail. Despite being used by many laboratories, only few sub-nanometer resolution structures have been reported so far using this technique, with the vast majority remaining at medium to low resolution (10-30 $\AA$ ). The first structure breaching the $5 \AA$ barrier was the structure of the immature HIV-1 CA-SP1, a capsid protein lattice assembled in presence of Bevirimat which was resolved to $3.9 \AA$ (Schur et al. 2016). The sample is very well suited for high-resolution SA - it is only around $200 \mathrm{~nm}$ thick, the virus-like particles contain a large number of the CA-SP1 protein, and the structure itself has C6 symmetry. It had been assumed that a large number of particles was essential in achieving the $3.9 \AA$ resolution. However, it was later shown that the same resolution could be achieved with less than $10 \%$ of particles using improved image processing methods and well-chosen subset of particles (Turonova et al. 2017). Recently, we have proposed a workflow to identify such subset of particles and introduced automatic removal of sub-optimal particles (Turonova et al. 2020). In this work we analyze and quantify the effect of sub-optimal particles on the final structure and its resolution. We propose sample-dependent techniques that explore different geometrical properties of structures to identify such particles and we show that removing the bad particles from the SA processing as early as possible leads to an improvement of structural features. Our geometrybased particle cleaning approach is not only suitable for regular arrangements of proteins but can be also applied to challenging samples involving large macromolecular complexes such as the human nuclear pore complex where achieving significant resolution using less particles is essential given the relatively low abundance of the protein complex of interest.

\section{References}

Schur, F. K. M. et al. An atomic model of hiv-1 capsid-sp1 reveals structures regulating assembly and maturation. Science 353, 506-508 (2016).

Turoňová, B., Schur, F. K., Wan, W. \& Briggs, J. A. Efficient 3d-ctf correction for cryo-electron tomography using novactf improves subtomogram averaging resolution to 3.4 Å. J. Struct. Biol. 199, 187195 (2017).

Turoňová, B., Hagen, W.J.H., Obr, M. et al. Benchmarking tomographic acquisition schemes for highresolution structural biology. Nat Commun 11, 876 (2020). 